\title{
Barrière de surface d'un semiconducteur en fonction de la température dans le cas de distributions complexes d'états. Calcul et exploitation
}

\author{
J. Bonnet, L. Soonckindt, J. M. Palau, H. Mansour et L. Lassabatère \\ Laboratoire d'études des Surfaces, Interfaces et Composants $\left({ }^{*}\right)$, \\ U.S.T.L., place Eugène Bataillon, 34060 Montpẹllier Cedex, France
}

(Reçu le 9 septembre 1983, révisé le 7 février 1984, accepté le 22 mars 1984)

\begin{abstract}
Résumé. - Pour interpréter les variations du travail de sortie des semiconducteurs avec la température on est conduit, à partir des différents modèles plausibles de distribution des états électroniques de surface à rechercher les variations de la barrière induite par ces états lorsque la température varie. Dans cet article, nous étudions de façon systématique les variations de la barrière de surface d'un semiconducteur en fonction de la température, dans le cas d'une distribution d'états complexes (deux états accepteurs discrets, un accepteur et un donneur discrets. un continuum uniforme, une distribution gaussienne). Nous montrons quel est alors le comportement de la surface, quelles caractéristiques peuvent être déterminées, et discutons de la façon de mettre en évidence l'existence de ces distributions.
\end{abstract}

\begin{abstract}
The results of a systematic study of the surface barrier height variations of a semiconductor versus temperature for complex surface state distributions (two discrete acceptor states, one discrete acceptor and one discrete donor, a uniform continuum distribution, a Gaussian distribution) are given. Then we show what is the behaviour of the surface, what surface properties can be determined and discuss how to make obvious the existence of these state distributions.
\end{abstract}

\section{Introduction.}

Un des buts fondamentaux, tant au plan théorique que technologique, de l'étude des surfaces semiconductrices est la détermination des états de surface situés dans la bande interdite. La connaissance des caractéristiques de tels états (nature, position, densité, distribution, attachement à une bande d'énergie) qui permet la détermination de la charge en surface $[1,2]$ est en effet nécessaire pour l'avancement de la recherche théorique mais aussi pour la physique des composants. Les techniques expérimentales les plus fréquemment ùtilisées sont des méthodes optiques reposant sur l'étude du rendement de photo-émission $[3,4]$ ou sur la recherche de transitions directes des états vers les bandes ou réciproquement. Dans le cas de la photoémission les états doivent être initialement remplis; la déconvolution des courbes caractéristiques des différents états de surface obtenus pour différents dopages permet d'obtenir des informations sur la forme et la distribution des états. La méthode n'est cependant pas très précise et ne permet pas d'exclure

(*) E.R.A. no 1022 , associée au C.N.R.S d'éventuelles perturbations, la mesure se faisant hors équilibre et en présence d'un agent extrêmement actif : le faisceau. Les autres méthodes optiques, telles que la modification du potentiel de surface (spectroscopie de photovoltage [5] ou de la conductivité [6]) sous l'action d'un rayonnement lumineux sont, quant à elles, difficiles au plan expérimental. Par ailleurs, pour préciser la distribution des états, on est le plus souvent conduit à en modifier l'occupation et pour cela étudier des échantillons de différents dopages.

L'utilisation de la température [7] comme paramètre permet dans une certaine mesure de s'affranchir de cet inconvénient. En effet quand la température varie le déplacement consécutif du niveau de Fermi contribue à un changement dans la charge des états. Si les conditions du vide sont telles que l'adsorption soit négligeable et si la réversibilité avec la température est parfaite, les mesures sont alors parfaitement significatives. Elles autorisent des spectroscopies basées sur l'étude en température du travail de sortie, du photovoltage ou de la conductivité. Elles sont toutefois délicates en raison de la dépendance de nombreux facteurs avec la température et impliquent 
des modélisations. Les résultats qu'elles fournissent sont néanmoins complémentaires de ceux obtenus par d'autres méthodes et ceci explique que les travaux anciens de P. Mark aient impulsé des recherches plus récentes comme celles de l'équipe de Mönch [8] par exemple dont les travaux reposent sur la mesure par la méthode non perturbatrice de Kelvin des variations des travaux de sortie avec la température.

Nous plaçant dans cette optique, nous avons déjà montré [9] que, dans le cas d'une distribution d'états représentable par une fonction de Dirac, la connaissance de l'évolution de $V_{\mathrm{s}}$ avec $T$ pouvait permettre d'accéder à la connaissance de la position énergétique et de la densité de l'état.

Nous nous proposons ici d'étudier le cas de distributions plus complexes dans le but de savoir comment les mettre en évidence et rechercher les informations que les variations de $V_{\mathrm{s}}$ avec $T$ apportent alors sur les caractéristiques des états. Comme l'on ne peut envisager d'étudier l'infinité de cas de distributions possibles, nous analysons ici les cas typiques suivants correspondant à des modèles fréquemment rencontrés dans les études tant théoriques qu'expérimentales des surfaces :

- modèle à deux états discrets de même type ou de types différents simultanément présents en surface [10-12],

- modèle de distributions d'états étendues (distribution gaussienne, continuum uniforme).

\section{Principes de base.}

1.1 Notations. - Nous utilisons les notations suivantes :

$$
\begin{aligned}
& A=2\left(\frac{2 m_{n} \pi k}{h^{2}}\right)^{3 / 2} T^{3 / 2} \frac{1}{N_{\mathrm{D}}-N_{\mathrm{A}}} \\
& B=\exp \left(\frac{-\alpha}{k}\right), \quad B=\left(\frac{-\alpha}{2 k}\right),
\end{aligned}
$$

$B=1$ suivant que l'état est attaché à la bande de valence, au milieu de la bande interdite ou à la bande de conduction lorsque $T$ varie.
$A^{\prime}=1 / A$

$B^{\prime}=1 / B$

$D_{\mathrm{S}}(E)=$ répartition en densité de l'état en fonction de l'énergie $E$

$E_{\mathrm{c}}, E_{\mathrm{v}}=$ énergie du minimum de la bande de conduction et du maximum de la bande de valence

$E_{\mathrm{F}}=$ énergie du niveau de Fermi

$E_{\mathrm{T}}=$ énergie d'un état de surface

$E_{0}=$ énergie centrale d'une distribution gaussienne

$f \pm=$ fonction de Fermi

$h=$ constante de Plank

$k=$ constante de Boltzmann

$m_{\mathrm{n}}=$ masse apparente des électrons

$N_{\mathrm{D}}-N_{\mathrm{A}}=$ densité de dopants en volume

$N_{\mathrm{s}}=$ densité d'un état de surface

$q=$ charge de l'électron

$Q_{\mathrm{SC}}=$ densité de la charge d'espace

$Q_{\text {Ss }}=$ densité de la charge superficielle

$T=$ température absolue

$V_{\mathrm{s}}=$ hauteur de la barrière en surface

$\alpha=$ coefficient de température du gap

$\varepsilon \quad=$ permittivité du matériau

$\sqrt{\gamma}=$ paramètre de précision d'une distribution gaussienne.

Les indices $\mathrm{A}, \mathrm{D}, \mathrm{O}, \mathrm{S}$ sont utilisés pour indiquer que le paramètre est relatif à un état accepteur ou donneur, que la valeur est considérée à $0 \mathrm{~K}$ et en surface.

Toutes les courbes sont tracées dans le cas d'un semiconducteur de type $\mathrm{n}$ tel que $\left(E_{\mathrm{c}}-E_{\mathrm{v}}\right)_{0}=1,4 \mathrm{eV}$; $\alpha=-34 \times 10^{-5} ; N_{\mathrm{D}}-N_{\mathrm{A}}=10^{23} \mathrm{~m}^{-3}$ sauf indication contraire.

1.2 EXPRESSION DE LA CHARGE EN VOLUME ET EN SURFACE. - Le calcul doit intégrer de nombreux paramètres tels que la structure des bandes du matériau et son évolution avec la température [13], le type de l'état, sa fonction de distribution, son évolution par rapport aux bandes avec $T$... [9].

Les courbes $Q_{\mathrm{SC}}$ tracées dans la suite de cet exposé seront calculées dans le cas le plus simple où la charge dans la zone de charge d'espace a pour expression

$$
\begin{aligned}
Q_{\mathrm{SC}}=\left[2 \varepsilon_{0} \varepsilon_{r} k T\left(N_{\mathrm{D}}-N_{\mathrm{A}}\right)\right]^{1 / 2} & \left\{\frac{4\left(2 m_{n} \pi k / h^{2}\right)^{3} T^{3} \exp \left[-\left(E_{\mathrm{c}}-E_{\mathrm{v}}\right)_{0} / k T\right]}{\left(N_{\mathrm{D}}-N_{\mathrm{A}}\right)^{2}} \times\right. \\
\times & \left.\exp \left(-\frac{\alpha_{0}}{k}\right)\left[\exp \left(-\frac{q V_{\mathrm{s}}}{k T}\right)+\frac{q V_{\mathrm{s}}}{k T}-1\right]+\exp \left(\frac{q V_{\mathrm{s}}}{k T}\right)-\frac{q V_{\mathrm{s}}}{k T}-1\right\}^{1 / 2} .
\end{aligned}
$$

L'expression de la charge dans les états de surface se déduit de l'expression générale

$$
Q_{\mathrm{SS}}= \pm \int_{\Delta E} D_{\mathrm{S}}(E) f \pm\left[\left(E-E_{\mathrm{f}}\right)_{\mathrm{s}}, T\right] \mathrm{d} E
$$

où le signe - est utilisé dans le cas d'états accepteurs, et le signe + dans le cas d'états donneurs. 
Pour un état accepteur discret, cette relation s'écrit

$$
Q_{\mathrm{SSA}}=\frac{-q N_{\mathrm{SA}}}{1+A B \exp \left[\frac{1}{k T}\left(E_{\mathrm{TA}}-E_{\mathrm{c}}\right)_{\mathrm{SO}}-q V_{\mathrm{s}}\right]} .
$$

Pour un état donneur discret

$$
Q_{\mathrm{SSD}}=\frac{q N_{\mathrm{SD}}}{1+A^{\prime} B^{\prime} \exp \left\{\left(-\frac{1}{k T}\right)\left[\left(E_{\mathrm{TD}}-E_{\mathrm{c}}\right)_{\mathrm{SO}}-q V_{\mathrm{s}}\right]\right\}} .
$$

1.3 PRINCIPE DE LA MÉTHODE. - A l'équilibre thermodynamique, la charge de $Q_{\mathrm{ss}}$ prise par l'état de surface doit être compensée par une charge en volume $Q_{\mathrm{SC}}$ égale et de signe opposé. Si les caractéristiques du volume et des états de surface sont parfaitement connues, on peut calculer les charges de $Q_{\mathrm{ss}}$ et $Q_{\mathrm{SC}}$ en fonction de la courbure des bandes $V_{\mathrm{s}}$ et de $T$. On peut donc tracer des réseaux $Q_{\mathrm{SS}}\left(V_{\mathrm{s}}\right)_{\mathrm{T}}$ et $Q_{\mathrm{SC}}\left(V_{\mathrm{s}}\right)_{\mathrm{T}}$. Pour chaque valeur de $T$, la condition $Q_{\mathrm{ss}}\left(V_{\mathrm{s}}\right)_{\mathrm{T}}+$ $Q_{\mathrm{SC}}\left(V_{\mathrm{s}}\right)_{\mathrm{T}}=0$ peut être calculé numériquement ou déduite graphiquement. Elle permet de déterminer la valeur de $V_{\mathrm{s}}$.

Pour éviter d'alourdir le texte par une analyse mathématique exhaustive, analyse qui conduit à des conclusions simples, mais dont la démarche est laborieuse, nous n'en présenterons que des résultats fondamentaux. Pour des raisons de simplification, nous nous sommes limités à l'étude d'un semiconducteur de type $\mathrm{n}$ en régime de déplétion-inversion. Dans la discussion, nous nous appuyons essentiellement sur le tracé des courbes qui résultent du calcul et illustrent de façon claire ses conclusions. La figure 1 représente dans un diagramme semilogarithmique le tracé de $\left|Q_{\mathrm{SC}}\left(V_{\mathrm{s}}\right)\right|_{\mathrm{T}}$ obtenu pour différentes valeurs du dopage. On note que la température intervient essentiellement lorsque le régime d'inversion est atteint, et que les réseaux représentatifs sont translatés vers le haut lorsque le dopage croît.

Les figures $2 a$ et $2 b$ représentent dans un diagramme semilogarithmique respectivement le tracé de la charge $\left|Q_{\mathrm{SS}}\left(V_{\mathrm{s}}\right)_{\mathrm{T}}\right|$ prise par un état accepteur discret pour différentes valeurs de la densité d'états et différentes valeurs de la position énergétique de l'état de surface. Les réseaux représentatifs sont translatés vers des valeurs plus importantes de la charge lorsque la densité d'états croît; ils sont translatés vers des valeurs plus grandes de $\left|V_{\mathrm{s}}\right|$ lorsque l'état s'éloigne de la bande de conduction. Chaque courbe $\log \left|Q_{\mathrm{ss}}\left(V_{\mathrm{s}}\right)\right|$ tend vers deux asymptotes, l'une horizontale, l'autre oblique. Plus le dopage est faible, plus le point d'intersection de ces asymptotes se trouve reporté vers des valeurs de $\left|V_{\mathbf{S}}\right|$ faibles.

Les variations de $V_{\mathrm{s}}$ avec $T$ dépendent donc de la position relative des réseaux, et en particulier du dopage du matériau et de la densité des états.

Nous avons montré [9] qu'un cas particulièrement intéressant était obtenu lorsque le recoupement des

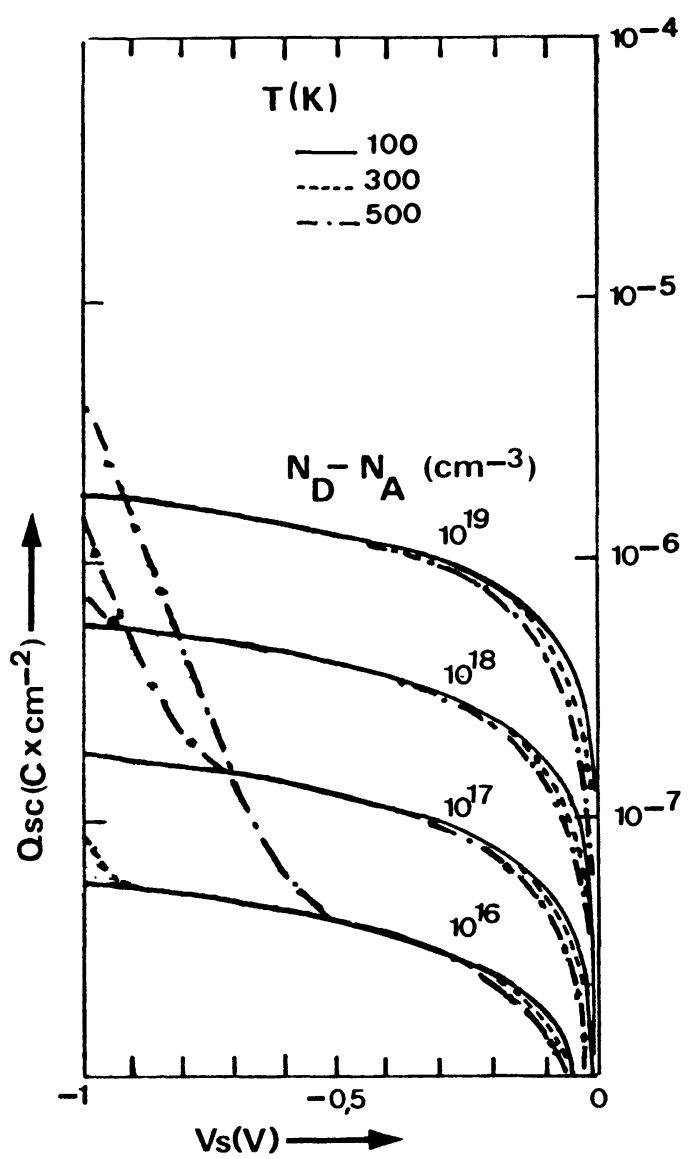

Fig. 1. - Charge dans la zone de charge d'espace en régime de déplétion-inversion en fonction de la courbure des bandes.

[Charge in the space charge region (with depletion-inversion layers) as a function of band bending.]

réseaux avait lieu dans la région où la charge dans l'état pouvait être calculée dans l'approximation de l'asymptote oblique. $V_{\mathrm{s}}(T)$ est alors une droite. Son extrapolation au zéro absolu permet la détermination de la position énergétique et sa pente celle de la densité de l'état.

\section{Association de deux états discrets.}

En supposant qu'il n'existe pas d'interaction entre les états, nous considérons successivement les deux distributions suivantes : deux états accepteurs A1 et A2 ou un état accepteur A et un état donneur D. 

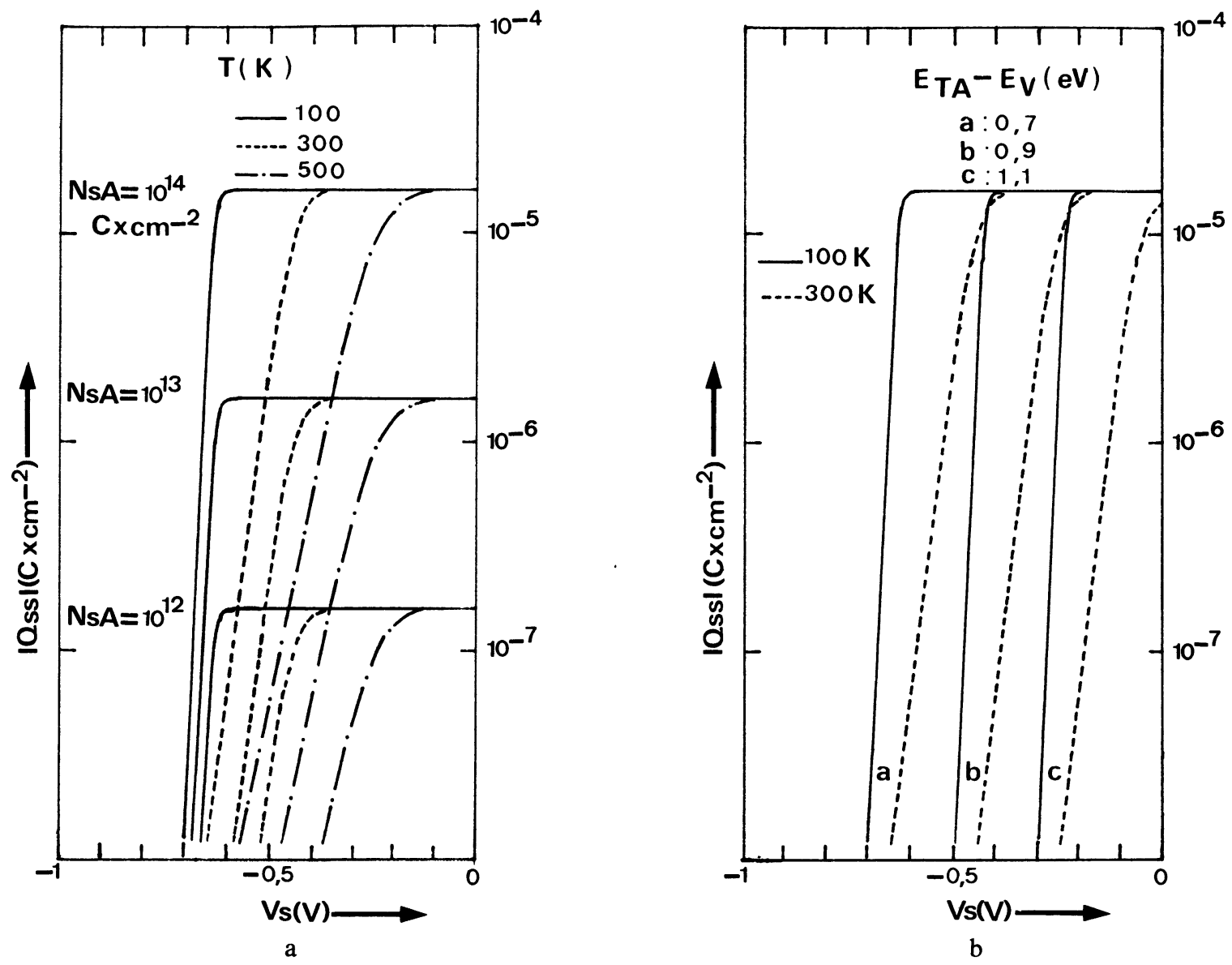

Fig. 2. - Charge d'un état accepteur discret en fonction de la courbure des bandes pour différentes températures. 2a : $E_{\mathrm{TA}}-E_{\mathrm{v}}=0,7 \mathrm{eV} .2 \mathrm{~b}: N_{\mathrm{SA}}=10^{14} \mathrm{~cm}^{-2}$.

[Charge of a discrete acceptor state as a function of band bending for various temperatures.]

Pour clarifier la discussion, nous supposons en outre les états situés à des positions énergétiques suffisamment différentes pour que les caractéristiques $Q_{\mathrm{ss}}\left(V_{\mathrm{s}}\right) T$ qui leur correspondent soient parfaitement distinctes dans le domaine de température étudié.

2. 1 DeUX ÉTATS ACCEPTEURS DISCRETS. - La charge totale en surface a pour expression

$$
\begin{aligned}
Q_{\mathrm{SS}}= & \frac{-q N_{\mathrm{SA} 1}}{1+A B \exp \left\{\frac{1}{k T}\left[\left(E_{\mathrm{TA} 1}-E_{\mathrm{c}}\right)_{\mathrm{SO}}-q V_{\mathrm{s}}\right]\right\}} \\
+ & \frac{-q N_{\mathrm{SA} 2}}{1+A B \exp \left\{\frac{1}{k T}\left[\left(E_{\mathrm{TA} 2}-E_{\mathrm{c}}\right)_{\mathrm{SO}}-q V_{\mathrm{s}}\right]\right\}}
\end{aligned}
$$

Désignons par A2 l'état le plus profond. On peut montrer que si $\mathrm{A} 1$ a une densité inférieure à celle de $\mathrm{A} 2$, la surface se comporte comme si seul l'état A2 était présent. L'état le moins profond se trouve donc entièrement masqué (Fig. 3a).

La figure $3 \mathrm{~b}$ illustre le cas où l'état $\mathrm{A} 1$ a une densité supérieure à celle de $\mathrm{A} 2$. La courbe $\left|Q_{\mathrm{ss}}\left(V_{\mathrm{s}}\right)\right|$ est alors caractérisée par deux parties horizontales et deux parties obliques, correspondant aux asymptotes horizontale et oblique des courbes représentatives de chacun des deux états.

Suivant la position relative des deux réseaux $\left|Q_{\mathrm{ss}}\left(V_{\mathrm{s}}\right)_{\mathrm{T}}\right|$ et $\left|Q_{\mathrm{sc}}\left(V_{\mathrm{s}}\right)_{\mathrm{T}}\right|$, la courbe $V_{\mathrm{s}}(T)$ obtenue peut permettre la détermination des paramètres relatifs à l'un ou l'autre des deux états.

La figure 4 qui montre les variations de $V_{\mathrm{s}}$ avec $T$ pour différentes densités de l'état le plus profond illustre ce point.

Lorsque les densités dans les deux états sont voisines, la variation de la charge séparant les deux asymptotes horizontales est d'un facteur 2. Reportée sur une échelle logarithmique, cette variation est faible. La caractéristique $\left|Q_{\mathrm{SS}}\left(V_{\mathrm{s}}\right)_{\mathrm{T}}\right|$ est très semblable à celle obtenue dans le cas où l'état le plus profond est aussi le plus dense ou seul. 

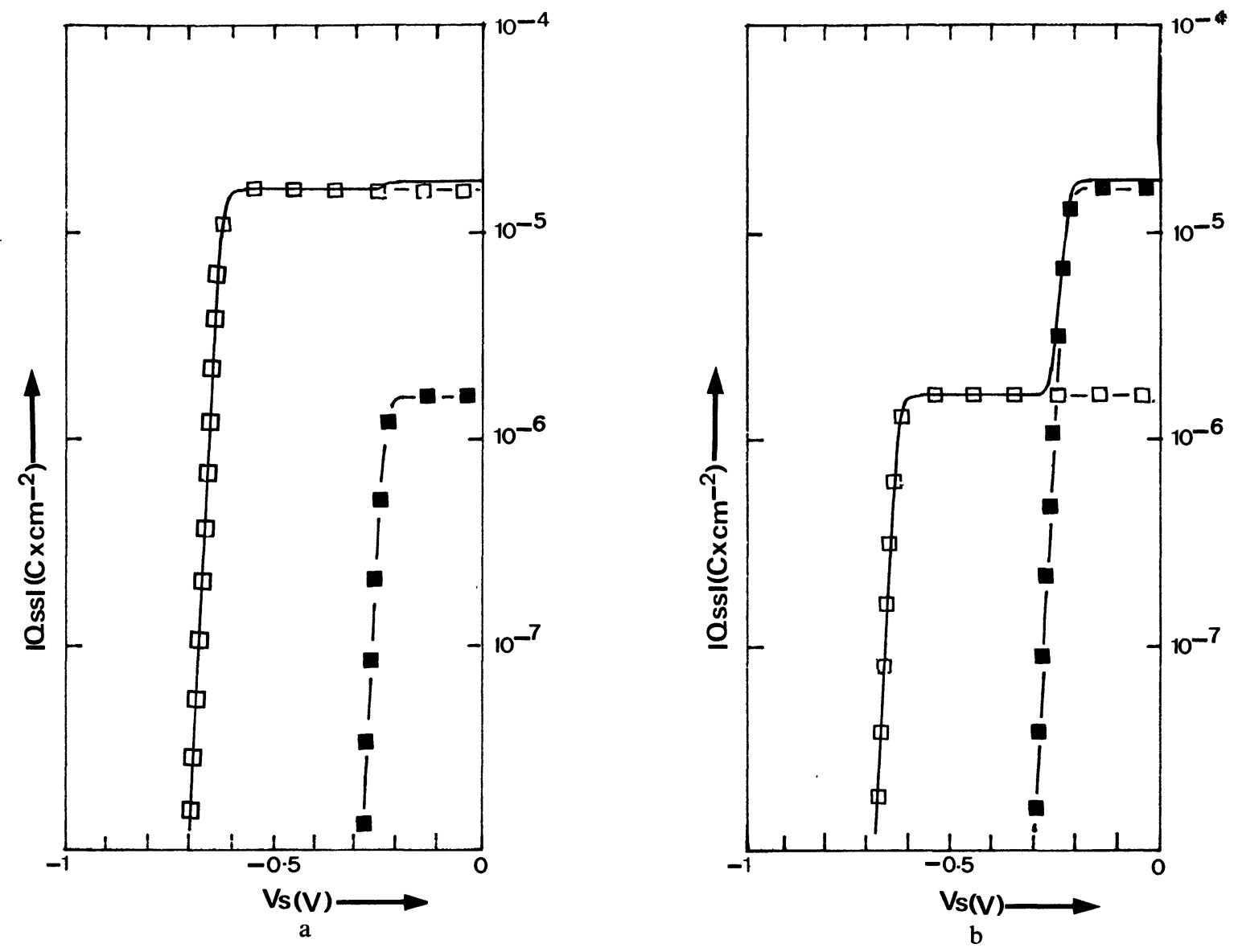

Fig. 3. - Cas de deux états discrets. Charge en surface en fonction de la courbure des bandes. $T=100 \mathrm{~K}$. — charge totale en surface, $\square \square$ charge en surface en présence du seul état $A 1, \square \square \square$ charge en surface en présence du seul état A2. 3a : L'état le plus profond (A2) a la plus faible densité. $E_{\mathrm{TA} 2}-E_{\mathrm{v}}=0,7 \mathrm{eV} ; N_{\mathrm{SA} 2}=10^{14} \mathrm{~cm}^{-2} ; E_{\mathrm{TA} 1}-E_{\mathrm{v}}=1,1 \mathrm{eV}$; $N_{\mathrm{SA} 1}=10^{13} \mathrm{~cm}^{-2} .3 \mathrm{~b}:$ L'état le moins profond (A1) a la plus faible densité. $E_{\mathrm{TA} 2}-E_{\mathrm{v}}=0,7 \mathrm{eV} ; N_{\mathrm{SA} 2}=10^{13} \mathrm{~cm}^{-2}$; $E_{\mathrm{TA} 1}-E_{\mathrm{v}}=1,1 \mathrm{eV} ; N_{\mathrm{SA} 1}=10^{14} \mathrm{~cm}^{-2}$.

[Case of two discrete states. Surface charge as a function of band bending. $T=100 \mathrm{~K}$.— whole surface charge, surface charge corresponding to the state A1, $\square \square \square$ surface charge corresponding to the state A2. 3a : The deeper state (A2) has the lowest density. $E_{\mathrm{TA} 2}-E_{\mathrm{v}}=0.7 \mathrm{eV} ; N_{\mathrm{SA} 2}=10^{14} \mathrm{~cm}^{-2} ; E_{\mathrm{TA} 1}-E_{\mathrm{v}}=1.1 \mathrm{eV} ; N_{\mathrm{SA} 1}=10^{13} \mathrm{~cm}^{-2} .3 \mathrm{~b}: \mathrm{The}$ less deep state (A1) has the lowest density. $E_{\mathrm{TA} 2}-E_{\mathrm{v}}=0.7 \mathrm{eV} ; N_{\mathrm{SA} 2}=10^{13} \mathrm{~cm}^{-2} ; E_{\mathrm{TA} 1}-E_{\mathrm{v}}=1.1 \mathrm{eV} ; N_{\mathrm{SA} 1}=$ $10^{14} \mathrm{~cm}^{-2}$.]

2.2 ETAT ACCEPTEUR ET ÉTAT DONNEUR DISCRETS SUR UN SEMICONDUCTEUR TYPE N. - La charge prise par la surface est

$$
\begin{aligned}
Q_{\mathrm{SS}}= & \frac{q N_{\mathrm{SD}}}{1+A^{\prime} B^{\prime} \exp \left\{-\frac{1}{k T}\left[\left(E_{\mathrm{TD}}-E_{\mathrm{c}}\right)_{\mathrm{SO}}-q V_{\mathrm{s}}\right]\right\}} \\
-\frac{q N_{\mathrm{SA}}}{1+A B \exp \left\{\frac{1}{k T}\left[\left(E_{\mathrm{TA}}-E_{\mathrm{c}}\right)_{\mathrm{SO}}-q V_{\mathrm{s}}\right]\right\}} & \text { (6) }
\end{aligned}
$$

Nous supposons ici que l'état donneur est assez profond pour ne pas mettre le semiconducteur de type $n$ en régime d'accumulation dans la gamme de température considérée. Deux possibilités sont envi- sageables suivant la position énergétique respective des deux états.

- Ou bien l'état donneur est plus profond que l'état accepteur de telle sorte que dans le domaine de température considéré les caractéristiques $\left|Q_{\mathrm{SSD}}\left(V_{\mathrm{s}}\right)\right|$ et $\left|Q_{\mathrm{SSA}}\left(V_{\mathrm{s}}\right)\right|$ restent nettement séparées (Fig. 5). L'intersection de $\left|Q_{\mathrm{SS}}\left(V_{\mathrm{s}}\right)_{\mathrm{T}}\right|$ et $\left|Q_{\mathrm{SC}}\left(V_{\mathrm{s}}\right)_{\mathrm{T}}\right|$ ne peut avoir lieu que dans la région de déplétion ou inversion. L'état accepteur intervient seul, et l'étude des courbes $V_{\mathrm{s}}(T)$ conduit à la détermination de ses caractéristiques. L'état donneur n'influe pas sur le comportement de la surface.

- Ou bien l'état donneur est moins profond que l'état accepteur. Il existe un domaine de valeurs de $V_{\mathrm{s}}$ dans lequel les caractéristiques de l'état s'inter- 


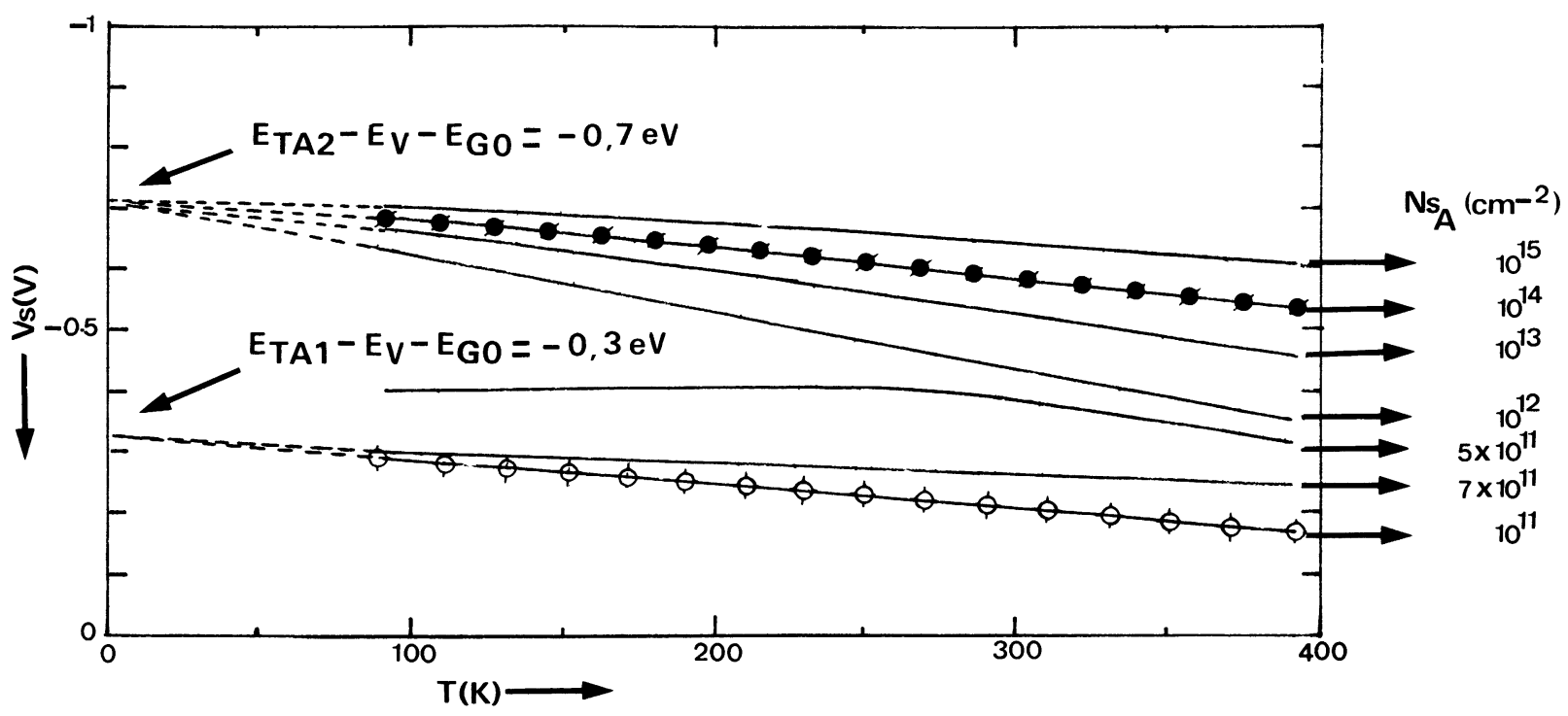

Fig. 4. - Courbure des bandes en fonction de la température lorsque deux états accepteurs sont présents en surface. $E_{\mathrm{TA} 1}-E_{\mathrm{v}}=1,1 \mathrm{eV} ; N_{\mathrm{SA} 1}=10^{14} \mathrm{~cm}^{-2} ; E_{\mathrm{TA} 2}-E_{\mathrm{v}}=0,7 \mathrm{eV} ; N_{\mathrm{SA} 2}$ paramètre. $O O O$ courbure calculée dans le cas où A1 est seul présent, 00 courbure calculée dans le cas où A2 est seul présent pour $N_{\mathrm{SA} 2}=10^{14} \mathrm{~cm}^{-2}$.

[Band bending corresponding to two acceptor surface states, as a function of temperature. $E_{\mathrm{TA} 1}-E_{\mathrm{v}}=1.1 \mathrm{eV} ; N_{\mathrm{SA} 1}=$ $10^{14} \mathrm{~cm}^{-2} ; E_{\mathrm{TA} 2}-E_{\mathrm{v}}=0.7 \mathrm{eV} ; N_{\mathrm{SA} 2}$ parameter. 000 band bending corresponding to the state A1, 00 band bending corresponding to the state A2 with $N_{\mathrm{SA} 2}=10^{-14} \mathrm{~cm}^{-2}$.]

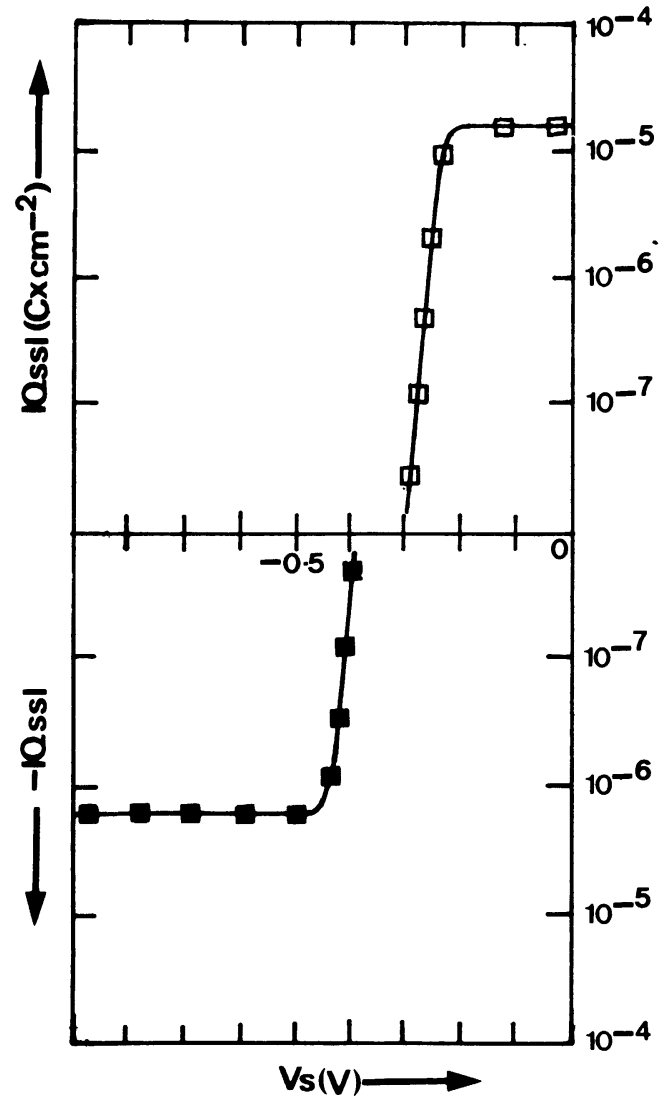

pénètrent. Comme le montre la figure 6 , on peut distinguer plusieurs zones distinctes de comportement numérotées de 1 à 5 , en fonction de la valeur de $V_{\mathrm{s}}$.
Fig. 5. - Charge en surface en présence d'un état accepteur et d'un état donneur discrets. Le donneur est plus profond que l'accepteur. $T=100 \mathrm{~K} . E_{\mathrm{TA}}-E_{\mathrm{v}}=1,1 \mathrm{eV} ; N_{\mathrm{SA}}=$ $10^{14} \mathrm{~cm}^{-2} ; E_{\mathrm{TD}}-E_{\mathrm{v}}=0,7 \mathrm{eV} ; N_{\mathrm{SD}}=10^{13} \mathrm{~cm}^{-2}$. charge totale en surface, $\square \square \square$ charge en présence du seul état accepteur, - @ charge en présence du seul état donneur.

[Surface charge corresponding to discrete acceptor and donor states. The donor is deeper than the acceptor. $T=$ $100 \mathrm{~K} . E_{\mathrm{TA}}-E_{\mathrm{v}}=1.1 \mathrm{eV} ; N_{\mathrm{SA}}=10^{14} \mathrm{~cm}^{-2} ; E_{\mathrm{TD}}-E_{\mathrm{v}}=$ $0.7 \mathrm{eV} ; N_{\mathrm{SD}}=10^{13} \mathrm{~cm}^{-2}$, whole surface charge, $\square \square \square$ charge corresponding to the acceptor state, charge corresponding to the donor state.]

- Zone 1. Cette région correspond aux faibles valeurs de $\left|V_{\mathrm{s}}\right|$. On peut montrer que

$$
Q_{\mathrm{ss}} \simeq q N_{\mathrm{SA}} \text {. }
$$

- Zone 2. Cette région correspond aux fortes valeurs de $\left|V_{\mathrm{s}}\right|$

$$
Q_{\mathrm{Ss}} \simeq q N_{\mathrm{SD}} \text {. }
$$

C'est l'asymptote horizontale qui correspond à l'état donneur.

- Zone 3. La valeur de $\left|V_{\mathrm{s}}\right|$ est suffisamment faible pour que l'état accepteur reste entièrement chargé, mais devient suffisamment importante pour que la charge de l'état donneur influe sur le compor- 

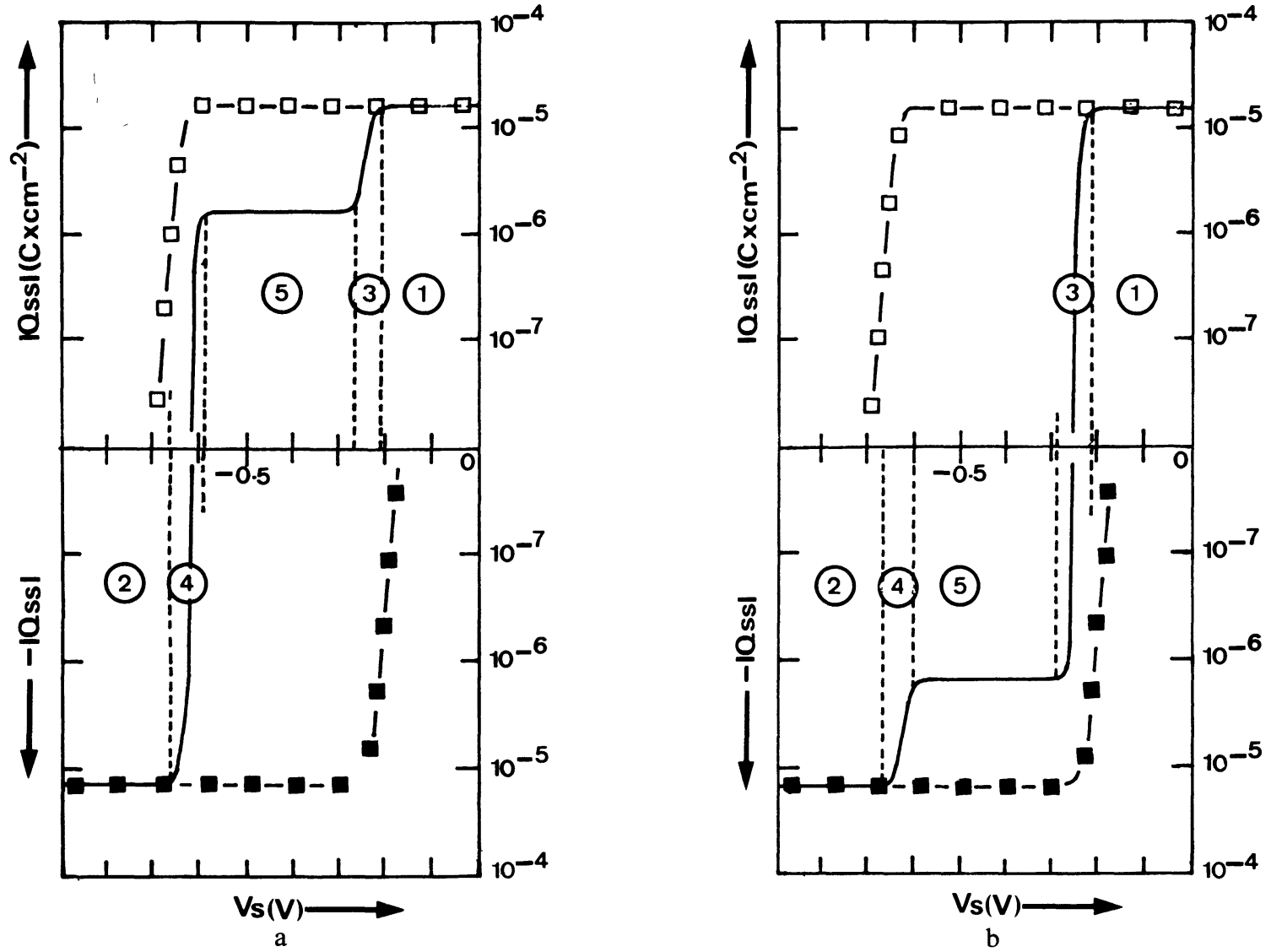

Fig. 6. - Charge en surface en présence d'un état accepteur et d'un état donneur discrets. L'accepteur est plus profond que le donneur. $T=100 \mathrm{~K} ; E_{\mathrm{TA}}-E_{\mathrm{v}}=0,7 \mathrm{eV} ; E_{\mathrm{TD}}-E_{\mathrm{v}}=1,1 \mathrm{eV}$. — charge totale en surface, $\square \square \square$ charge en présence du seul état accepteur, - a charge en présence du seul état donneur, 1 à $5:$ différentes zones de comportement, $6 \mathrm{a}$ : l'accepteur est plus dense que le donneur, $N_{\mathrm{SA}}=10^{14} \mathrm{~cm}^{-2} ; N_{\mathrm{SD}}=9 \times 10^{13} \mathrm{~cm}^{-2}, 6 \mathrm{~b}$ : le donneur est plus dense que l'accepteur $/ N_{\mathrm{SA}}=9 \times 10^{13} \mathrm{~cm}^{-2} ; N_{\mathrm{SD}}=10^{16} \mathrm{~cm}^{-2}$.

[Surface charge corresponding to discrete acceptor and donor states. The acceptor is deeper than the donor. $T=100 \mathrm{~K}$, whole surface charge, $\square \square \square$ charge corresponding to the acceptor state, $\square$ - charge corresponding to the donor state, 1 to 5 : various behaviours, $6 \mathrm{a}:$ the acceptor density if higher than the donor one, $N_{\mathrm{SA}}=10^{14} \mathrm{~cm}^{-2} ; N_{\mathrm{SD}}=9 \times$ $10^{13} \mathrm{~cm}^{-2}, 6 \mathrm{~b}:$ the donor density is higher than the acceptor one, $N_{\mathrm{SA}}=9 \times 10^{13} \mathrm{~cm}^{-2} ; N_{\mathrm{SD}}=10^{16} \mathrm{~cm}^{-2}$.]

tement de la surface :

ou

$$
Q_{\mathrm{SS}}=-q N_{\mathrm{SA}}+\frac{q N_{\mathrm{SD}}}{1+A^{\prime} B^{\prime} \exp \left\{\left(-\frac{1}{k T}\right)\left[\left(E_{\mathrm{TD}}-E_{\mathrm{c}}\right)_{\mathrm{SO}}-q V_{\mathrm{s}}\right]\right\}}
$$

$$
Q_{\mathrm{SS}}=\left(-q N_{\mathrm{SA}}+q N_{\mathrm{SD}}\right)+\frac{-q N_{\mathrm{SD}}}{1+A B \exp \left\{\frac{1}{k T}\left[\left(E_{\mathrm{TD}}-E_{\mathrm{c}}\right) S_{\mathrm{O}}-q V_{\mathrm{s}}\right]\right\}} .
$$

$\mathrm{Si}\left|q N_{\mathrm{SA}}\right| \gg\left|q N_{\mathrm{SD}}\right|, Q_{\mathrm{SS}} \simeq-q N_{\mathrm{SA}}(10 \mathrm{bis})$.

La surface se comporte comme si l'état accepteur était seul présent. $\mathrm{Si}$

$$
\left|q N_{\mathrm{SA}}\right| \ll \frac{\left|q N_{\mathrm{SD}}\right|}{1+A^{\prime} B^{\prime} \exp \left\{\left(-\frac{1}{k T}\right)\left[\left(E_{\mathrm{TD}}-E_{\mathrm{c}}\right)_{\mathrm{SO}}-q V_{\mathrm{s}}\right]\right\}}
$$




$$
Q_{\mathrm{SS}} \simeq \frac{q N_{\mathrm{SD}}}{1+A^{\prime} B^{\prime} \exp \left\{\left(-\frac{1}{k T}\right)\left[\left(E_{\mathrm{TD}}-E_{\mathrm{c}}\right)_{\mathrm{SO}}-q V_{\mathrm{s}}\right]\right\}}
$$

La surface se comporte comme si l'état donneur était seul présent. Si $\left|q N_{\mathrm{SA}}\right| \simeq\left|q N_{\mathrm{SD}}\right|$

$$
Q_{\mathrm{SS}} \simeq \frac{-q N_{\mathrm{SD}}}{1+A B \exp \left\{\frac{1}{k T}\left[\left(E_{\mathrm{TD}}-E_{\mathrm{c}}\right)_{\mathrm{SO}}-q V_{\mathrm{s}}\right]\right\}} .
$$

La forme de l'expression de $Q_{\mathrm{ss}}$ est celle correspondant à un état accepteur, qui aurait les caractéristiques (densité et position énergétique) de l'état donneur.

- Zone 4. Elle représente le cas symétrique de la zone 3 : suivant la densité de chacun des deux états, la charge en surface se comporte comme si l'état donneur était seul présent $\left(\left|q N_{\mathrm{SD}}\right| \gg\left|q N_{\mathrm{SA}}\right|\right)$, comme si l'état accepteur était seul présent,

$$
\left|q N_{\mathrm{SD}}\right| \ll \frac{\left|q N_{\mathrm{SA}}\right|}{1+A B \exp \left\{\left(-\frac{1}{k T}\right)\left[\left(E_{\mathrm{TD}}-E_{\mathrm{c}}\right)_{\mathrm{SO}}-q V_{\mathrm{s}}\right]\right\}}
$$

ou comme si l'on était en présence d'un état donneur ayant les caractéristiques électriques de l'état accepteur $\left(\left|q N_{\mathrm{SA}}\right| \simeq\left|q N_{\mathrm{SD}}\right|\right)$.

- Zone 5. La valeur de $\left|V_{\mathrm{s}}\right|$ est ici telle que l'état accepteur et l'état donneur sont entièrement chargés,

$$
Q_{\mathrm{SS}}=q N_{\mathrm{SD}}-q N_{\mathrm{SA}} .
$$

La représentation de $\left|Q_{\mathrm{Ss}}\left(V_{\mathrm{s}}\right)_{\mathrm{T}}\right|$ présente donc une troisième partie horizontale qui, suivant la valeur relative de $\left|q N_{\mathrm{SD}}\right|$ et $\left|q N_{\mathrm{SA}}\right|$ peut correspondre à une charge positive ou à une charge négative en surface.

Dans la région des charges en surfaces négatives, qui correspond au cas où le semiconducteur de type $\mathrm{n}$ est en régime de déplétion-inversion, on peut donc obtenir plusieurs types de représentations suivant les densités relatives dans chacun des deux états.

Si l'état accepteur est beaucoup plus dense que l'état donneur, la surface se comporte comme si l'accepteur était le seul présent.

Si l'état donneur est plus dense que l'accepteur, la caractéristique $\left|Q_{\mathrm{ss}}\left(V_{\mathrm{s}}\right)\right|$ présente une asymptote horizontale $Q_{\mathrm{ss}}=-q N_{\mathrm{SA}}$ et une asymptote oblique dont l'équation correspond à celle d'un état accepteur ayant les caractéristiques de l'état donneur (Fig. 6). L'état accepteur révèle la présence du donneur en surface, et les courbes $V_{\mathrm{s}}(T)$ interprétées dans cette dernière région conduisent à la détermination de la position énergétique et de la densité du donneur.

Si l'état donneur est moins dense que l'état accepteur, à la représentation précédente s'ajoutent une partie horizontale et une partie oblique, correspondant à des valeurs plus faibles de la charge (Fig. 6a). La représentation est semblable à celle obtenue dans le cas de la présence de deux états accepteurs discrets. L'équation de la partie horizontale fait apparaître la valeur de la différence de densité entre les deux états. L'équation de la partie oblique fait intervenir les caractéristiques de l'état donneur.

Le réseau de caractéristiques $V_{\mathrm{s}}(T)$ tracé figure 7 dans le cas d'un état accepteur de densité variable, plus profond que l'état donneur de densité constante, illustre l'influence des densités relatives dans chacun des deux états.

\section{Distributions étendues.}

Il est probable que les états réels ont une distribution étendue et il est donc important de connaître l'influence de cette distribution, tant par son extension que par sa forme, sur le comportement de la surface [14]. C'est pourquoi nous examinerons successivement le cas d'un continuum uniforme d'états et celui d'une distribution gaussienne.

3.1 ContinuUm UNIFORME D'ÉTATS. - On se place dans l'hypothèse d'états accepteurs de densité $N_{\mathrm{SA}}$, distribués uniformément en énergie dans la bande $\left[E_{\mathrm{T} 2}-E_{\mathrm{T} 1}\right]\left(E_{\mathrm{T} 2}<E_{\mathrm{T} 1}\right)$. La charge dans les états a pour expression

$$
\begin{aligned}
& Q_{\mathrm{SSA}}=-q N_{\mathrm{SA}}+\frac{q N_{\mathrm{SA}}}{E_{\mathrm{T} 2}-E_{\mathrm{T} 1}} \frac{k T}{q} \times \\
& \times \log \frac{1+A B \exp \left\{\frac{1}{k T}\left[\left(E_{\mathrm{T} 1}-E_{\mathrm{c}}\right)_{\mathrm{SO}}-q V_{\mathrm{s}}\right]\right\}}{1+A B \exp \left\{\frac{1}{k T}\left[\left(E_{\mathrm{T} 2}-E_{\mathrm{c}}\right)_{\mathrm{SO}}-q V_{\mathrm{s}}\right]\right\}}
\end{aligned}
$$

Les courbes 2 à 5 de la figure $8 \mathrm{a}$ représentent à $T=100 \mathrm{~K}$ les variations de $\left|Q_{\text {SSA }}\right|$ en fonction de la courbure des bandes, pour des valeurs croissantes de l'écart $E_{\mathrm{T} 2}-E_{\mathrm{T} 1}, E_{\mathrm{T} 2}$ restant fixe. La courbe 1 donne les variations de la charge dans un état discret de même densité, situé à l'énergie $E_{\mathrm{T} 2}$ :

- pour les faibles valeurs de $\left|V_{\mathrm{s}}\right|$, les courbes tendent vers la même asymptote horizontale, correspondant à l'état entièrement chargé, 


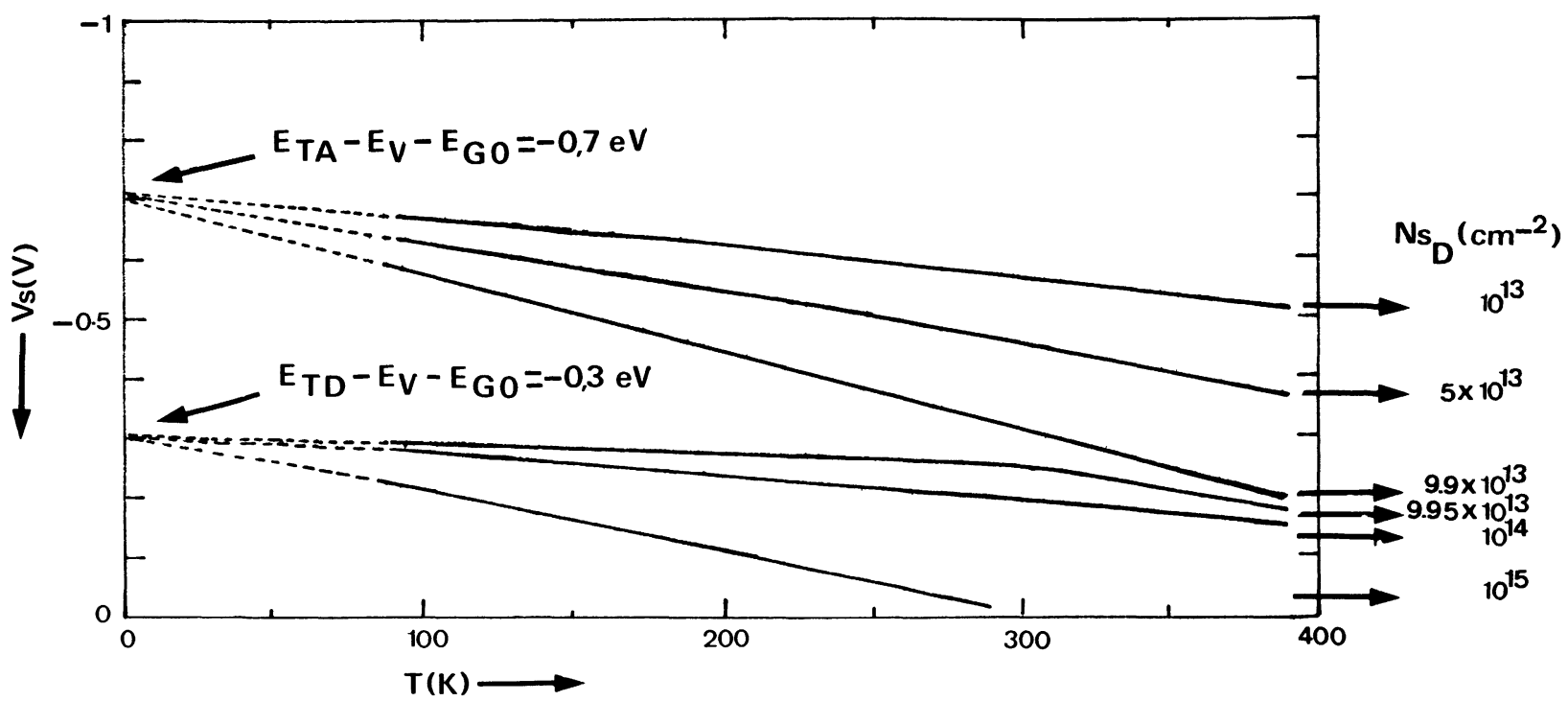

Fig. 7. - Courbure des bandes en fonction de la température lorsqu'un état accepteur et un état donneur moins profond sont simultanément présents en surface. $E_{\mathrm{TA}}-E_{\mathrm{v}}=0,7 \mathrm{eV} ; N_{\mathrm{SA}}=10^{14} \mathrm{~cm}^{-2} ; E_{\mathrm{TD}}-E_{\mathrm{v}}=1,1 \mathrm{eV} ; N_{\mathrm{SD}}$ paramètre.

[Band bending as a function of temperature when an acceptor state and a less deep donor state exist simultaneously on the surface. $E_{\mathrm{TA}}-E_{\mathrm{v}}=0.7 \mathrm{eV} ; N_{\mathrm{SA}}=10^{14} \mathrm{~cm}^{-2} ; E_{\mathrm{TD}}-E_{\mathrm{v}}=1.1 \mathrm{eV} ; N_{\mathrm{SD}}:$ parameter.]
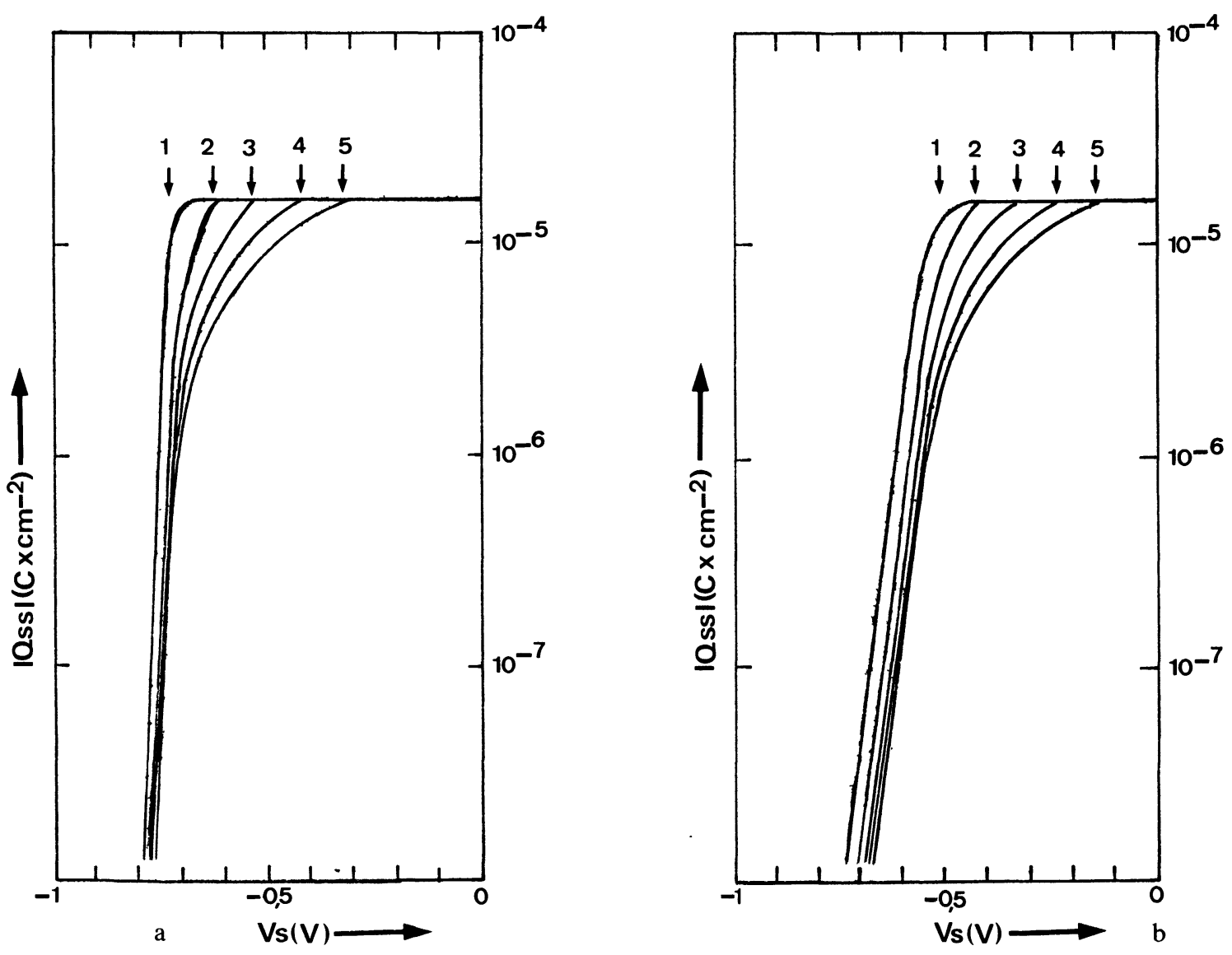

Fig. 8. - Charge en surface en présence d'un continuum accepteur uniforme. $E_{\mathrm{T} 2}-E_{\mathrm{v}}=0,6 \mathrm{eV}:$ limite basse du continuum. $E_{\mathrm{T} 1}-E_{\mathrm{v}}$ : paramètre limite haute du continuum, $N_{\mathrm{S}}=10^{14} \mathrm{~cm}^{-2}$. 1 - état discret, $2-E_{\mathrm{T} 1}-E_{\mathrm{v}}=0,7 \mathrm{eV}, 3-E_{\mathrm{T} 1}-$ $E_{\mathrm{v}}=0,8 \mathrm{eV}, 4-E_{\mathrm{T} 1}-E_{\mathrm{v}}=0,9 \mathrm{eV}, 5-E_{\mathrm{T} 1}-E_{\mathrm{v}}=1 \mathrm{eV}, 8 \mathrm{a}: T=100 \mathrm{~K}, 8 \mathrm{~b}: T=300 \mathrm{~K}$.

[Surface charge corresponding to a uniform acceptor continuum. $E_{\mathrm{T} 2}-E_{\mathrm{v}}=0.6 \mathrm{eV}$, low limit of the continuum. $E_{\mathrm{T} 1}-$ $E_{\mathrm{v}}=$ parameter, high limit of the continuum. $N_{\mathrm{S}}=10^{14} \mathrm{~cm}^{-2}$.] 
- pour les faibles valeurs du remplissage de l'état, elles tendent sensiblement vers la même asymptote oblique que l'état discret,

- loin de l'asymptote oblique, les tracés s'écartent d'autant plus de celui de l'état discret que l'écart $E_{\mathrm{T} 1}-E_{\mathrm{T} 2}$ est plus important, et que la température est plus élevée, comme le montre la comparaison avec les courbes de la figure $8 \mathrm{~b}$, tracées à $T=300 \mathrm{~K}$,

- à charge constante, l'écart entre les caractéristiques est proportionnel à la température, comme dans le cas d'un état discret (Figs. 9a et 9b).

Si les caractéristiques de l'état et du semiconducteur sont telles que le recoupement des réseaux $\log \left|Q_{\mathrm{SS}}\left(V_{\mathrm{s}}\right)_{\mathrm{T}}\right|$ et $\log \left|Q_{\mathrm{SC}}\left(V_{\mathrm{s}}\right)_{\mathrm{T}}\right|$ se produise lorsque l'état est entièrement chargé, rien ne distingue l'effet d'un continuum de celui d'un état discret. La courbure des bandes en surface ne dépend que de la densité de l'état.

$\mathrm{Si}$ le recoupement des deux réseaux a lieu lorsqu'une faible fraction de l'état se trouve chargée, les variations de $V_{\mathrm{s}}$ avec $T$ qui dépendent essentiellement de l'écart entre les asymptotes obliques, restent linéaires.
Cet écart étant un peu plus important dans le cas d'un continuum que dans celui d'un état discret, les variations de $V_{\mathrm{s}}$ avec $T$ sont plus importantes lorsque la distribution est étendue. Elles correspondent à celles que l'on obtiendrait avec un état discret de densité plus faible. Comme les asymptotes tendent vers la même limite à basse température, l'extrapolation des courbes $V_{\mathrm{s}}(T)$ correspondantes à $0 \mathrm{~K}$ conduit à la détermination de la limite basse $E_{\mathrm{T} 2} \mathrm{du}$ continuum.

Ici encore, rien ne permet de distinguer expérimentalement le cas du continuum de celui de l'état discret.

La distinction ne peut être envisagée que dans la région intermédiaire entre les deux cas précédents, région qui est plus étendue dans le cas du continuum puisque le vidage de l'état commence dès que la courbure des bandes devient suffisante pour amener la limite haute $E_{\mathrm{T} 1}$ au voisinage du niveau de Fermi. Les figures $10 \mathrm{a}$ et $10 \mathrm{~b}$ qui représentent les variations de $V_{\mathrm{s}}$ avec $T$ dans les deux types de distributions, et pour différentes densités d'état, illustrent la discussion
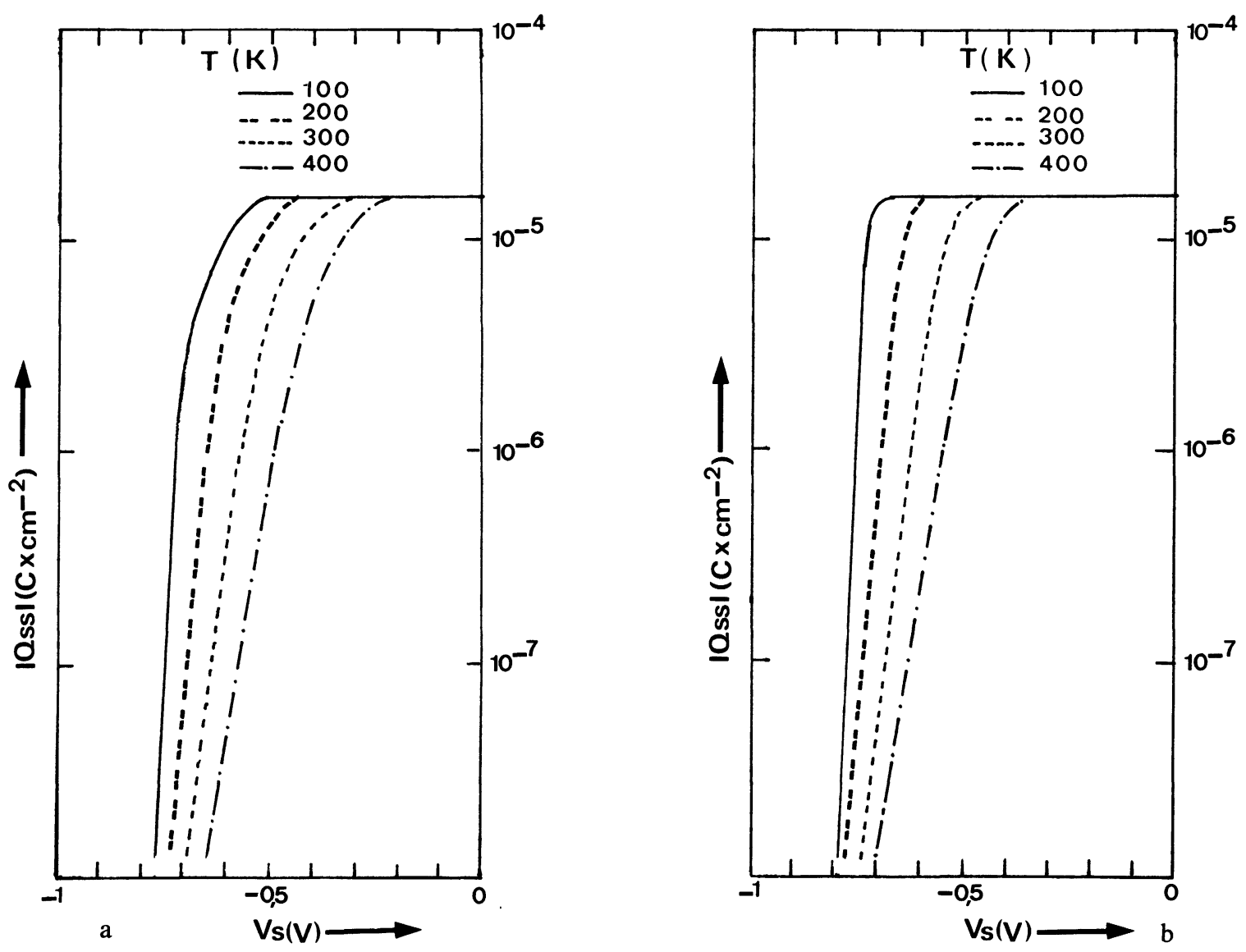

Fig. 9. - Charge en surface pour différentes températures. 9a : en présence d'un continuum accepteur uniforme, $E_{\mathrm{T} 1}-$ $E_{\mathrm{v}}=0,8 \mathrm{eV} ; E_{\mathrm{T} 2}-E_{\mathrm{v}}=0,6 \mathrm{eV} ; N_{\mathrm{S}}=10^{14} \mathrm{~cm}^{-2}$. $9 \mathrm{~b}$ : en présence d'un état accepteur discret. $E_{\mathrm{TA}}-E_{\mathrm{v}}=0,6 \mathrm{eV}$; $N_{\mathrm{SA}}=10^{14} \mathrm{~cm}^{-2}$.

[Surface charge corresponding to various temperatures. 9a : when a uniform acceptor continuum exists. $E_{\mathrm{T} 1}-E_{\mathrm{v}}=$ $0.8 \mathrm{eV} ; E_{\mathrm{T} 2}-E_{\mathrm{v}}=0.6 \mathrm{eV} ; N_{\mathrm{S}}=10^{14} \mathrm{~cm}^{-2} .9 \mathrm{~b}$ : when a discrete acceptor state exists. $E_{\mathrm{TA}}-E_{\mathrm{v}}=0.6 \mathrm{eV} ; N_{\mathrm{SA}}=10^{14} \mathrm{~cm}^{-2}$.] 


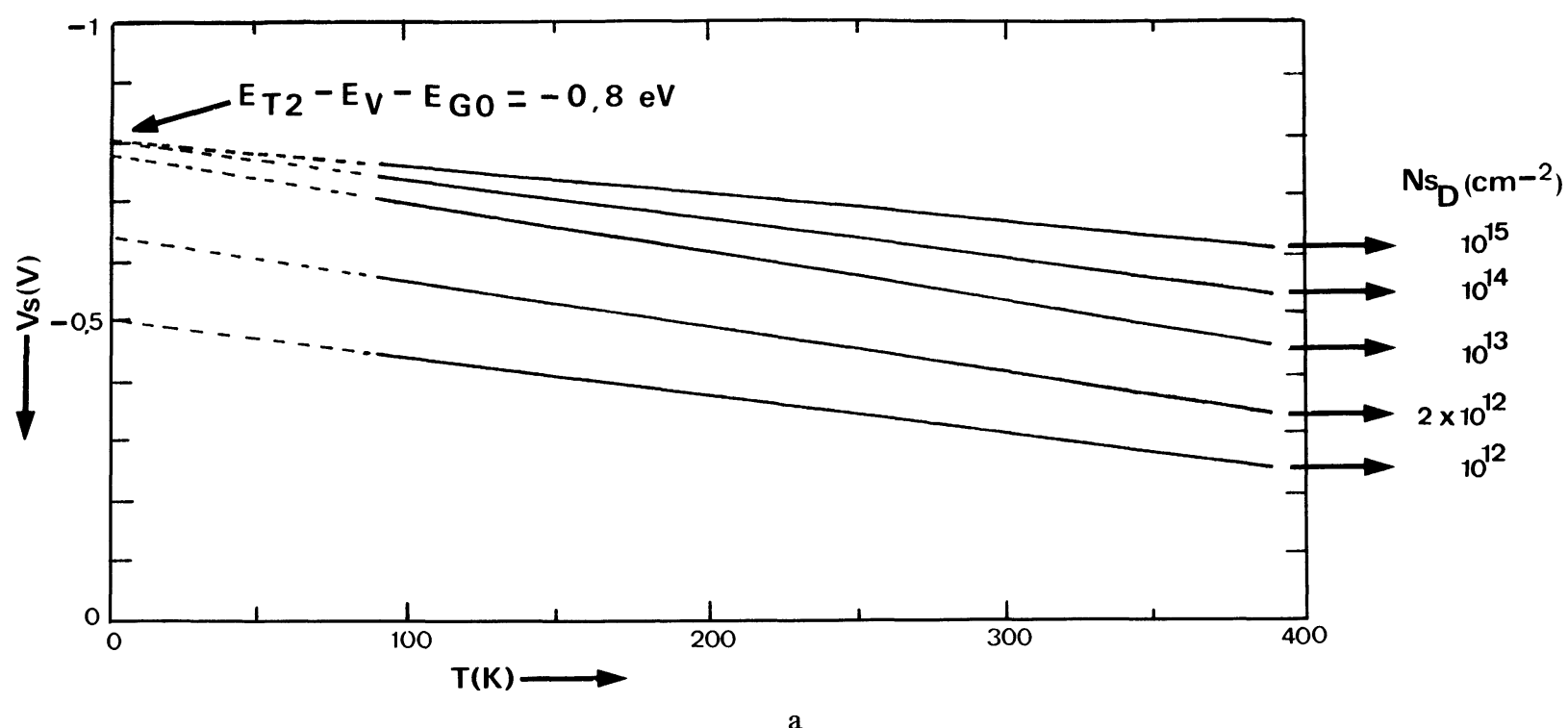

a

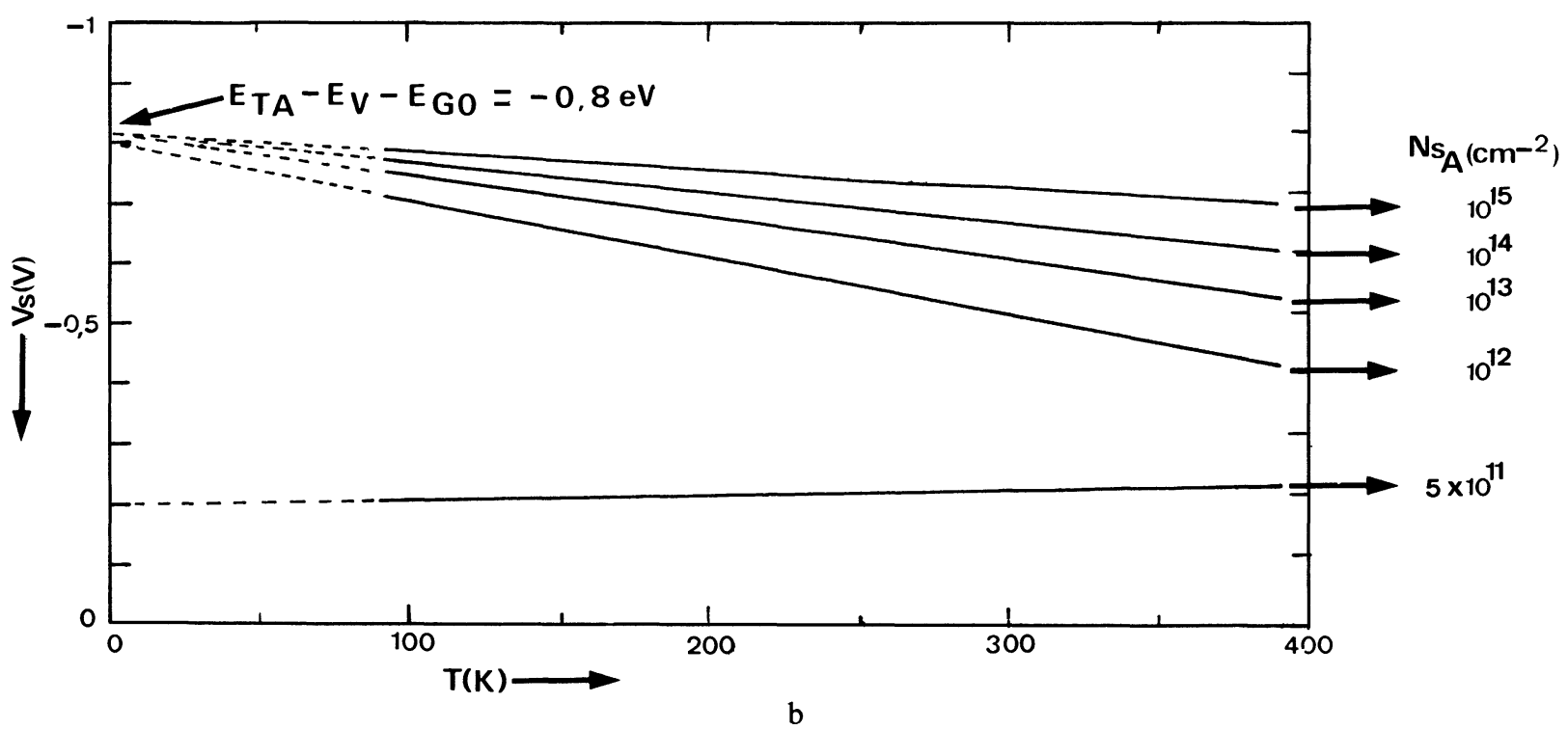

Fig. 10. - Courbure des bandes en fonction de la température. 10a : lorsqu'un continuum accepteur est présent en surface. $E_{\mathrm{T} 1}-E_{\mathrm{v}}=1 \mathrm{eV}: E_{\mathrm{T} 2}-E_{\mathrm{v}}=0,6 \mathrm{eV}$. $10 \mathrm{~b}:$ lorsqu'un état accepteur discret est présent en surface, $E_{\mathrm{TA}}-E_{\mathrm{v}}=0,6 \mathrm{eV}$.

[Band bending as a function of temperature. $10 \mathrm{a}$ : when an acceptor continuum exists, $E_{\mathrm{T} 1}-E_{\mathrm{v}}=1 \mathrm{eV} ; E_{\mathrm{T} 2}-E_{\mathrm{v}}=0.6 \mathrm{eV}$. $10 \mathrm{~b}$ : when a discrete acceptor state exists, $E_{\mathrm{TA}}-E_{\mathrm{v}}=0.6 \mathrm{eV}$.]

précédente. Elles montrent en outre, que le recoupement n'a lieu dans la région intermédiaire que pour une gamme étroite de densités, ce qui rend en général le résultat difficilement exploitable sur le plan expérimental.

La mise en évidence peut toutefois être faite lorsque l'on peut contrôler expérimentalement la densité d'états, c'est-à-dire essentiellement dans les études d'adsorption de gaz ou de dépôts métalliques [15-17]. On peut alors tracer une courbe $V_{\mathrm{s}}\left(N_{\mathrm{s}}\right)_{\mathrm{T}}$ représentant, à température constante, les variations de la courbure des bandes de surface en fonction de la densité d'états, comme représenté figure 11 où les courbes $\mathrm{a}, \mathrm{b}, \mathrm{c}$ ont été calculées à température fixe, en considérant respectivement le cas d'un état discret (courbe a) et le cas d'un continuum d'étendue paramétrée (courbes b et c).

Comme cela a été discuté plus haut, rien ne distingue ces courbes entre elles aux faibles valeurs de $N_{\mathrm{s}}$, et la différence reste faible aux fortes valeurs de $N_{\mathrm{s}}$. Par contre, l'extension de la région intermédiaire varie en fonction de la position énergétique de la limite haute du continuum; elle peut permettre par ajustage d'apprécier l'extension de l'état. 


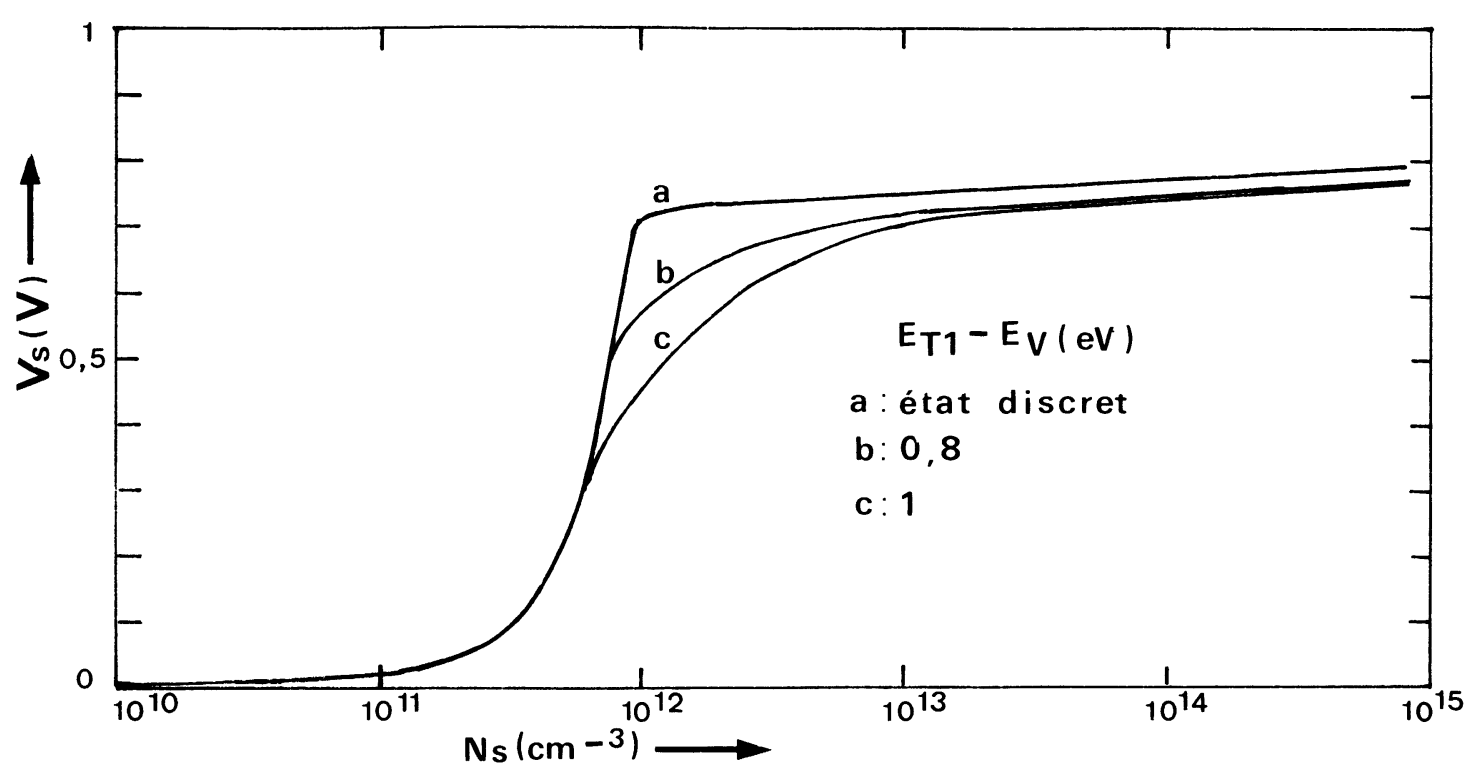

Fig. 11. - Courbure des bandes en présence d'un continuum accepteur de densité variable. Limite basse fixe $E_{\mathrm{T} 2}-E_{\mathrm{v}}=$ $0,6 \mathrm{eV}$. Limite haute $E_{\mathrm{T} 1}$ variable. Comparaison avec le cas d'un état discret.

[Band bending corresponding to an acceptor continuum of variable density. The low limit is fixed : $E_{\mathrm{T} 2}-E_{\mathrm{v}}=0.6 \mathrm{eV}$. The high limit $E_{\mathrm{T} 1}$ is variable. Comparison with the case of a discrete state.]

3.2 Distribution de Gauss. - Dans le cas d'une distribution gaussienne de paramètre de précision $\sqrt{\gamma}$, et centrée sur l'énergie $E_{0}$, la charge prise par l'état accepteur s'écrit :

$$
Q_{\mathrm{SSA}}=-\frac{q}{k T} \sqrt{\frac{\gamma}{\pi}} q N_{\mathrm{SA}} \int_{E_{\mathrm{v}}}^{E_{\mathrm{c}}} \frac{\mathrm{d} E}{\left[1+\exp \left\{\frac{B}{T}\left(E-E_{\mathrm{GO}}-V_{\mathrm{s}}\right)_{A, B}\right\}\right]\left[\exp \gamma\left\{\frac{B}{T}\left(E-E_{0}\right)^{2}\right\}\right]}
$$

Les courbes 1 des figures $12 \mathrm{a}, \mathrm{b}, \mathrm{c}$ représentent la charge prise par l'état, centré sur l'énergie $E_{0}$ fixe, en fonction de la courbure des bandes $V_{\mathrm{s}}$, respectivement pour des valeurs du paramètre $\gamma$ de $0,1,0,05$ et 0,01 . Les courbes 2 , tracées en encadré sur ces mêmes figures représentent la distribution correspondante en fonction de l'énergie. Les courbes 3 représentent la charge prise par un état discret de même densité, dont la position énergétique a été ajustée pour suivre au mieux les courbes 1 aux faibles valeurs de remplissage de l'état.

Comme dans le cas d'un continuum uniforme, la courbe relative à la gaussienne s'écarte d'autant plus de celle correspondant à un état discret que la distribution est plus étendue. Pour les bandes d'états peu étendues, on peut trouver un état discret dont la charge varie sensiblement de la même façon que pour la distribution, aux faibles valeurs de remplissage. Cet état est d'autant plus éloigné du centre $E_{0}$ de la gaussienne, que la distribution est plus étendue.
On peut empiriquement le situer à une énergie $E_{\mathrm{T}}$ correspondant à un remplissage de $10 \%$ de la distribution, tant que cette dernière n'est pas très étendue.

Les courbes 1, 2, 3 de la figure 13 représentent les variations de la charge dans un état en fonction de la courbure des bandes à trois températures différentes. Comme dans le cas d'un état discret, l'écart entre les parties obliques de la représentation est proportionnel à $T$. Les courbes $V_{\mathrm{s}}(T)$ sont donc linéaires et leur exploitation conduit à la détermination de $E_{\mathrm{T}}$ et à une évaluation de la densité de l'état.

\section{Conclusion.}

Le tableau de la figure 14 résume les informations apportées sur les états de surface par le relevé des courbes $V_{\mathrm{s}}(T)$ effectuées sur un semiconducteur de type $\mathrm{n}$, lorsque le recoupement des courbes $Q_{\mathrm{SC}}\left(V_{\mathrm{s}}\right)_{\mathrm{T}}$ se fait avec l'asymptote oblique de $\log \left|Q_{\mathrm{ss}}\left(V_{\mathrm{s}}\right)_{\mathrm{T}}\right|$. 

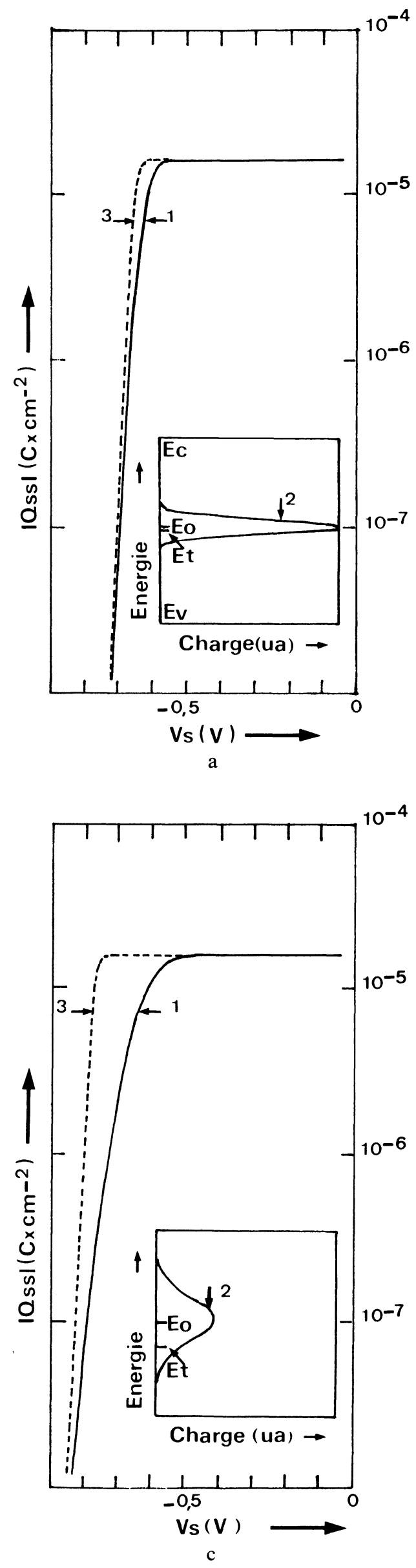

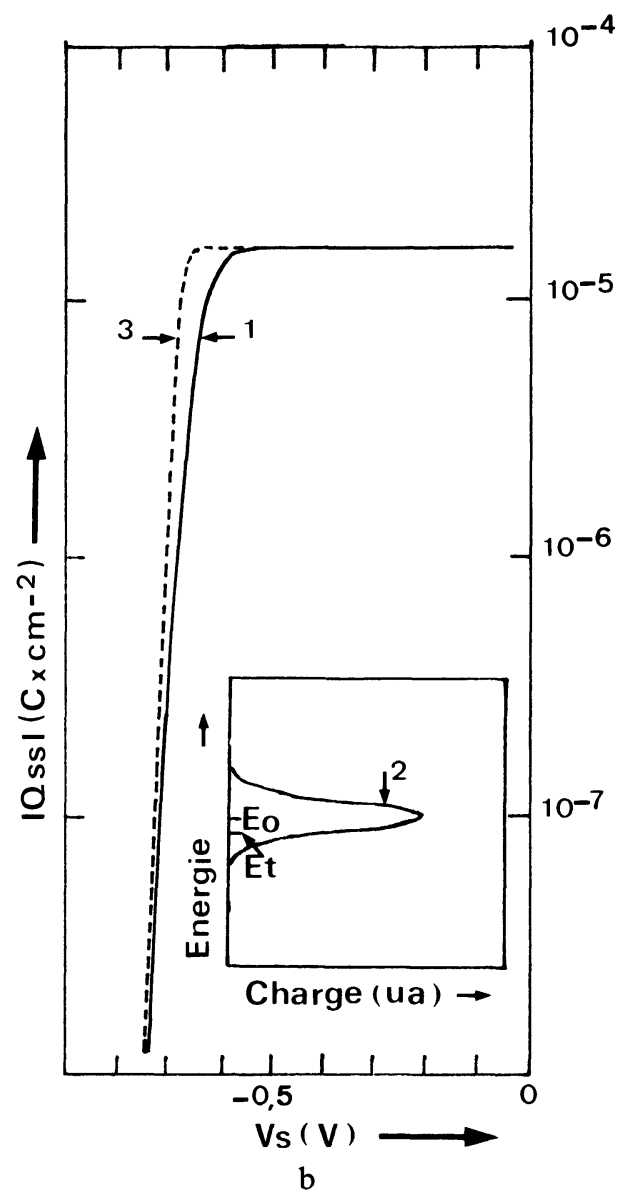

Fig. 12. - Charge en surface en présence d'une bande d'états accepteurs de répartition gaussienne centrée sur $E_{0}=0,7 \mathrm{eV}$ et de densité $N_{\mathrm{S}}=10^{14} \mathrm{~cm}^{-2}, T=100 \mathrm{~K}$. Charge dans un état accepteur discret de même densité, dont la position énergétique $E_{\mathrm{T}}$ a été ajustée pour faire coïncider les asymptotes obliques des représentations. En encadré : densité d'états en fonction de l'énergie. 12a : $\gamma=0,1 ; E_{\mathrm{T}}-E_{\mathrm{v}}=0.69 \mathrm{eV}, 12 \mathrm{~b}: \gamma=0,05 ; E_{\mathrm{T}}-E_{\mathrm{v}}=0,65 \mathrm{eV}$, $12 \mathrm{c}: \gamma=0,01 ; E_{\mathrm{T}}-E_{\mathrm{v}}=0,55 \mathrm{eV}$.

[Surface charge corresponding to a Gaussian surface state distribution centred on $E_{0}=0.7 \mathrm{eV}$. Density : $N_{\mathrm{S}}=$ $10^{14} \mathrm{~cm}^{-2} . T=100 \mathrm{~K}$. --- Surface charge in a discrete acceptor state $E_{\mathrm{T}}$ of same density; the energy of $E_{\mathrm{T}}$ has been adjusted to have the oblique asymptotes fitted. In frame : state density versus energy.] 


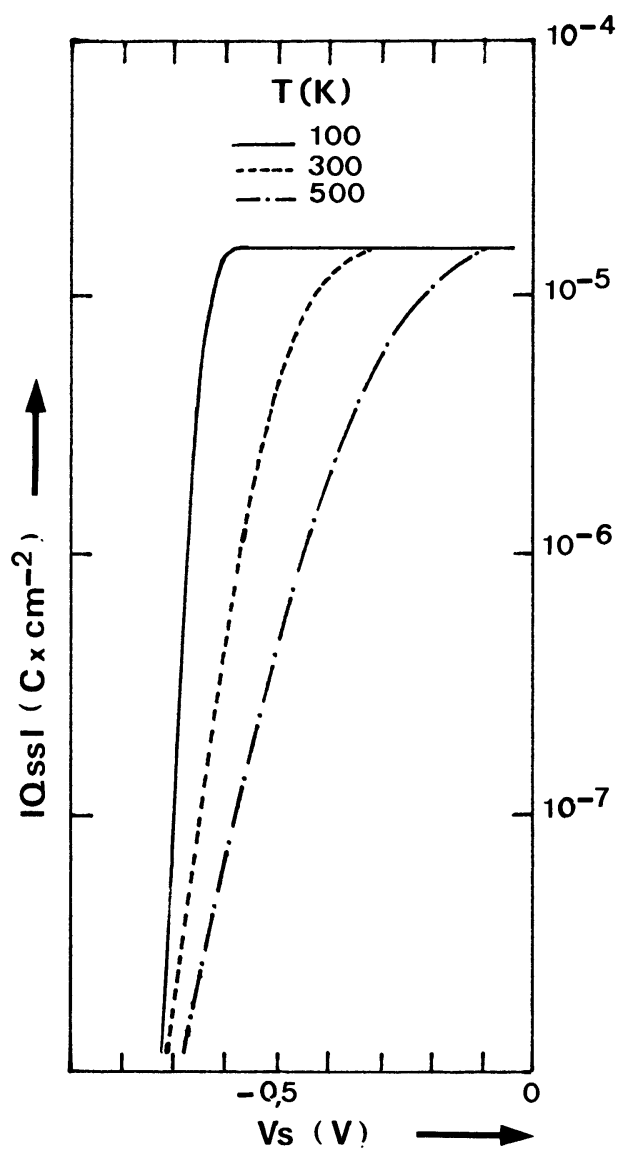

Fig. 13. - Charge en surface en présence d'une bande d'états accepteurs de répartition gaussienne centrée sur $E_{0}=0,7 \mathrm{eV}$, et de densité $N_{\mathrm{S}}=10^{14} \mathrm{~cm}^{-2}$. Influence de la température.

[Surface charge corresponding to an acceptor continuum with a Gaussian distribution centred on $E_{0}=0.7 \mathrm{eV}$. Surface density $N_{\mathrm{S}}=10^{14} \mathrm{~cm}^{-2}$. Influence of temperature.]
Dans ces conditions la courbure des bandes dépend peu des fluctuations de densité des états de surface ou des variations du dopage. Nous plaçant dans l'hypothèse de quelques cas simples très fréquemment utilisés pour étudier l'ancrage du niveau de Fermi lors de l'interaction métal-semiconducteur, nous avons montré que la surface se comporte en température comme si un état accepteur était seul présent. Les variations de $V_{\mathrm{s}}$ avec $T$ sont linéaires, comme dans le cas d'un état accepteur discret. Le comportement électrique de la surface en température dépend peu de la forme de la distribution énergétique des états. La figure 14 précise, pour les différentes distributions, la position énergétique du niveau de Fermi en surface obtenue par extrapolation au zéro absolu des courbes $V_{\mathrm{s}}(T)$. Les relevés expérimentaux des variations de $V_{\mathrm{s}}(T)$, dont on déduit la charge et la nature des états, permettent de déterminer cette position et d'en tirer des distributions possibles des états de surface. Certaines d'entre elles peuvent être ensuite rejetées en jouant sur des paramètres comme le type, le dopage, la densité des états. Il suffit, par exemple, d'utiliser pour cela les propriétés des relevés topographiques qui permettent de sélectionner des zones de surface dont les états aient la même position énergétique mais des densités très différentes au lieu de travailler sur des échantillons de différents dopages. De plus, de façon inverse, on peut partir de distributions obtenues par d'autres méthodes (spectroscopie de photo-émission, de photovoltage) et chercher à confirmer ou compléter des résultats par l'étude en température. Enfin, l'utilisation de la méthode en présence d'un continuum permet, en déplaçant le niveau de Fermi, d'avoir une idée de son extension et des densités d'état.

\section{Bibliographie}

[1] Many, A., Goldstein, Y., Grover, N. B., Semiconductor Surfaces (North Holland, Amsterdam) 1965.

[2] LiLe, D. L., Surf. Sci. 34 (1973) 337.

[3] SPICER, W. E., J. Physique 34 (1973) C6.

[4] Sebenne, C., Bolmont, P., Guichar, G. and BalKANSKI, M., Phys. Rev. B 12 (1975) 3280.

[5] Gatos, H. C., Lagowski, J., J. Vac. Sci. Technol. 10 $n^{\circ} 1$ (1973) 130.

[6] Chiarotti, G., Nannarone, S., Pastore, R., ChaRAdIA, P., Phys. Rev. B 4 (1971) 3398.

[7] MARK, P., Surf. Sci. 25 (1971) 192.

[8] Mönch, W., Clemens, H. J., J. Vac. Sci. Technol. 16 (5) (1979) 1238

[9] Bonnet, J., SoOnckindt, L., Lassabatère, L., Thin Solid Films 91 (1982) 283

[10] Palau, J. M., Ismail, A., Testemale-Vieujot, E. and Lassabatìre, L., Proceedings of the 16th International Conference on the Physics of semi- conductors, 6/10 septembre 1982, Montpellier, France, North Holland (Amsterdam).

[11] LeVIne, J. D., J. Vac. Sci. Technol. 6 (4) (1969) 549.

[12] Spicer, W. E., Chye, P. W., Skeath, P. R., Su, C. Y. and LindaU, I., J. Vac. Sci. Technol. 16 (5) (1979) 1422.

[13] Bonnet, J., Soonckindt, L. et Lassabatère, L., Surf. Sci. 91 (1980) 283.

[14] Palau, J. M., Testemale, E., Ismail, A. and LassaBATÈRE, L., J. Vac. Sci. Technol. 21 (1) (1982) 6.

[15] Spicer, W. E., Chye, P. W., Garner, C. M., Lindau, I. and Pianetta, P., Surf. Sci. 86 (1979) 763.

[16] Bonnet, J., Soonckindt, L. and Lassabatère, L., Proceedings of the International conference on Solid Surface and the third European conference on Surface Science, sept. 22-26 1980, Cannes, France. Sté Française du Vide.

[17] Bolmont, D., Chen, P. and Sebenne, C. A., Surf. Sci. 117 (1982) 417. 


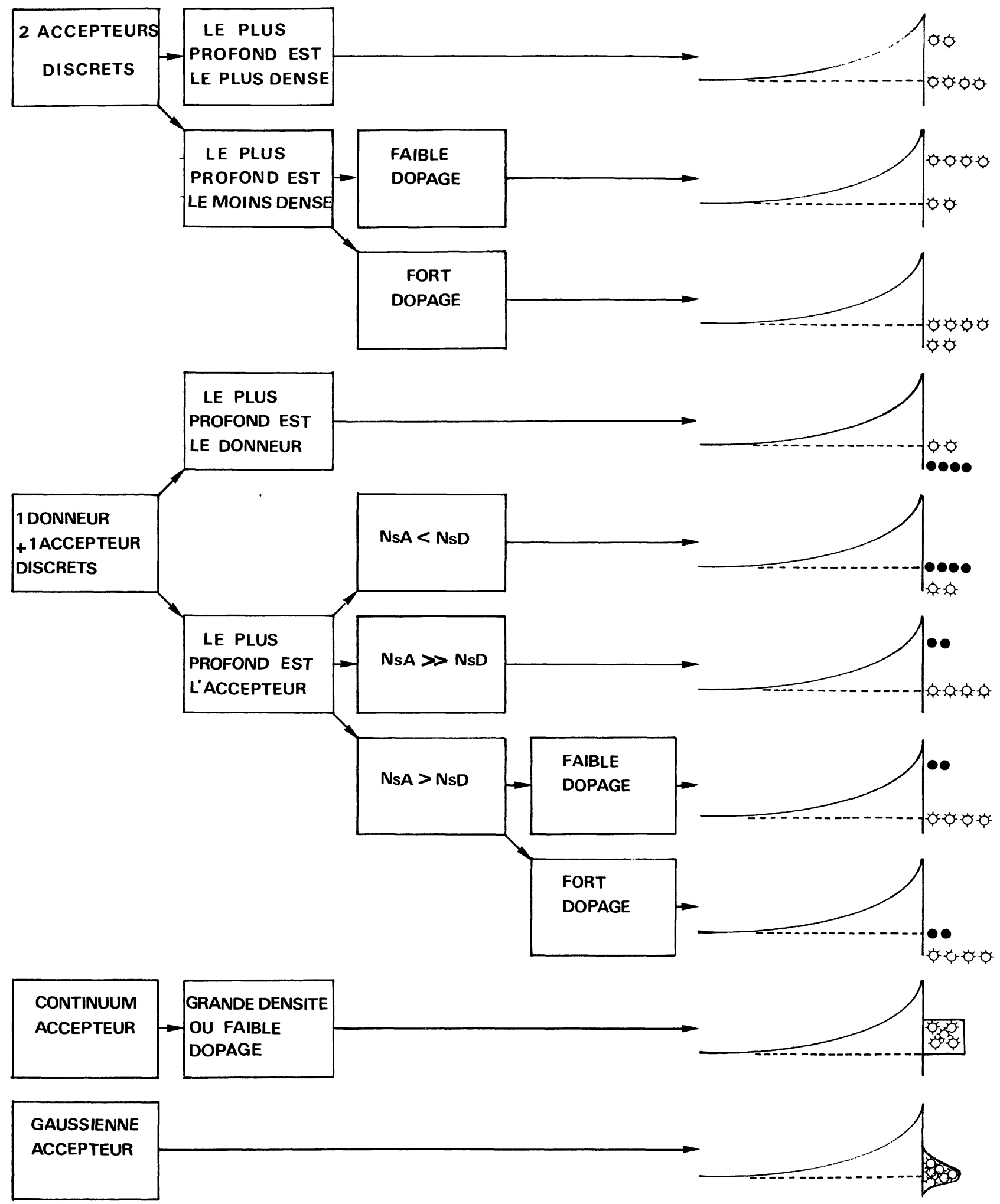

Fig. 14. - Rappel schématique de la signification de la mesure de la courbure des bandes à basse température, pour les

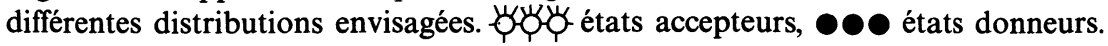

[Recall of the significance of the band bending at low temperature for different distributions. $\not \not \psi$-acceptor states, donor states.] 